\title{
Method for calculating the power of a rotor-screw machines
}

\author{
Valeriy A. Lebedev ${ }^{1, *}$, Georgy V. Serga ${ }^{2}$, Irina $V$. Davydova ${ }^{1}$, Tatiana $V$. Atoyan ${ }^{1}$, \\ Irina G. Koshlyakova ${ }^{1}$, and Alexander $V$. Gordienko ${ }^{1}$ \\ ${ }^{1}$ Don State Technical University, 344000 Rostov-on-Don, Gagarin square 1, Russia \\ ${ }^{2}$ I.T. Trubilin Kuban State Agrarian University, 350044 Krasnodar, Kalinina street 13, Russia
}

\begin{abstract}
Features of parts machining in rotor-screw technological systems are presented. It is shown that the mass of the load, consisting of the processed parts and the granulated working medium, is located along the internal perimeter of the screw rotor asymmetrically to the axis of its rotation. In this regard, there are difficulties in designing the rotor-screw machines drive, and in the rationale for its optimal power. A technique is proposed for calculating the drive power of rotor-screw machines, which makes it possible to carry out a techno-economic assessment study of their applicability for finishing-hardening machining of parts in machinebuilding production. The technique is illustrated on the basis of justifying the drive power of a rotor-screw machine for hardening the details of St3 steel in a screw rotor mounted from 9 octahedra, steel balls are used as a working medium.
\end{abstract}

\section{Features of Rotor-Screw Process Systems for Machining Parts}

Finishing-hardening technologies are known to ensure the machining of parts in rotor-screw technological systems with oscillations amplitude in the loading mass in the working organ - screw rotor from 10 to $1000 \mathrm{~mm}$ and higher [1,2].

Figure 1 shows a general view of a rotor screw machine for finishing hardening of parts, which works as follows. In the rotating screw rotor 4, mounted from the octahedral elements, through the loading means 8 , the granulated working medium and the parts to be processed are continuously loaded.

\section{Main content and results of work}

Figure 1 shows a diagram of the machine based on a screw rotor mounted from octahedral elements. Such a machine includes a frame 1 made in the form of a welded frame. The main drive 2, four roller bearings 3 are fixed to the frame. The screw rotor 4 is provided with two rims 5 and 6 , which are supported by roller bearings with flanges of support 3 fixed on two shafts 7. A loading means 8, a hopper 9 for reception of the processed parts and a hopper 10 for receiving working media. Above the hopper 10, in the screw rotor 5 ,

\footnotetext{
* Corresponding author: va.lebidev@yandex.ru
} 
holes 11 are provided for separating from the processed parts of the working medium particles and their transfer beyond the screw rotor to the hopper 10. To increase the speed of the longitudinal movement of the load masses, a tension spring 12 is fixed within the screw rotor 4.

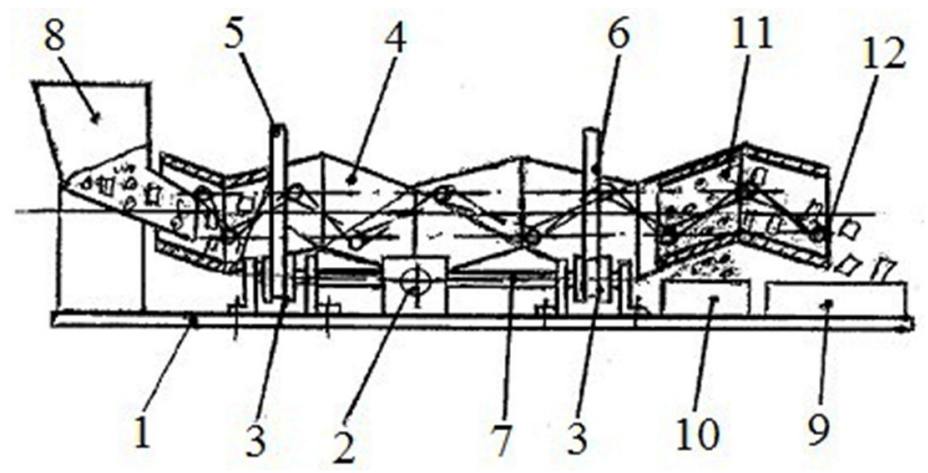

Fig. 1. Scheme of the machine based on a screw rotor, mounted from octahedral elements: 1 -bed; 2 - drive the main movement; 3 - roller bearings; 4 - screw rotor; 5 and 6 -rims; 7 - two shafts on which the roller bearings 3 are fixed; 8 means for loading; 9 - bunker for receiving processed parts; 10 - bunker for reception of working media; 11 - openings for the removal of working media; 12 - tension spring.

The screw rotor (Figure 3) is assembled from sections 1 made of equilateral six, eight, ten, etc. triangles 2 connected by their faces 3 . As a result of joining the sections to each other, a multi-pass surface is formed along the perimeter of the screw rotor with pronounced multi-pass continuous broken screw lines directed towards each other. In Figure 2, the thickened lines show one of the three right screw lines 4-5-6-7-8-9-10 of the main direction and one of the three left screw lines 11-12-8-13-14-5-15 of the opposite direction with step equal to the step of the main direction. Such a screw rotor is characterized by the fact that the cross-sectional area along the length in one step of the screw lines varies twice in each section, for example from sections assembled from six triangles in the form of octahedra from hexagon to triangle and again to hexagon (Figure 2, sections $\mathrm{A}-\mathrm{A}, \mathrm{B}-\mathrm{B}, \mathrm{B}-\mathrm{B}$ ). One step contains six sections with a variable cross-sectional area, which disrupts the steady-state motion of the load masses in the screw rotor.
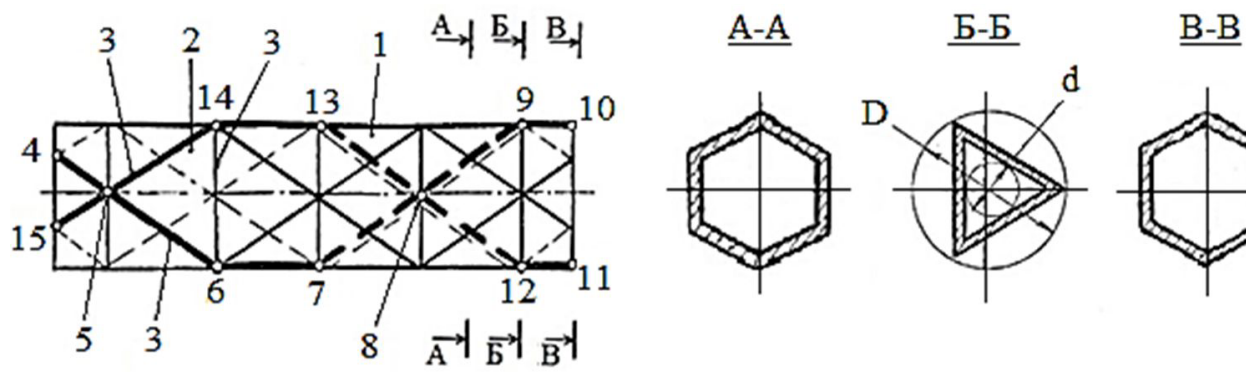

Fig. 2. The working body of the machine for finishing-cleaning and parts hardening processing (OZiUO) ( $\mathrm{a}$ - front view, $\mathrm{b}$ - top view), with a representation of the cross sections of the load in the screw rotor.

Screw lines along the outer surface of the screw rotor have the same designation of positions with the corresponding grooves on the inner surface, while $\mathrm{d}$ - is the diameter of the screw rotor cross section, $D$ - is the outer diameter of the screw rotor outer periphery. 
The machine for finishing-cleaning and reinforcing parts machining (OZiUO) works as follows. In the rotating screw rotor 4 , through the loading 8 , the working medium and the parts to be processed are continuously loaded. When the screw rotor 4 rotates, the working medium and the processed parts move along the screw grooves inside the screw rotor with a large amplitude and are discharged to the hopper for machined parts receiving 9 and the working medium through the holes 11 to the hopper 10. The amplitude of the movement of the machined parts and working medium particles is determined not only by the diameter of the cross-section of the screw rotor 4 , but also by the frequency of its rotation.

When the screw rotor 4 rotates, the media particles and machined parts are grasped by the faces of the inner screw surface and they rise upward in the direction of rotation and move towards the discharge side. Upon reaching a certain height, under the influence of gravitational forces and the formed angle of a natural slope, the particles of working media and the processed parts move towards each other at certain angles and to the walls of the rotating screw rotor 4 and move towards the unload. Since the broken inner surface of the screw rotor 4 is continuous, the process of moving the subsequent portions of the machined parts and working media is continuous as well that rise upwards and fall downwards, move continuously at different angles. Since the inner surface of the screw rotor (Figure 2) is mounted from equilateral triangles 2, which are located at different angles not only to each other but also to the axis of screw rotor rotation, each portion of the particles of working media and machined parts move along their direction vector towards unloading, which greatly intensifies the process of interaction of working media and machined parts with each other and with the walls of the screw rotor 4, increases the intensity of machining of parts and expands technological capabilities. Since due to the broken shape of the inner surface of the screw rotor 4 the range of changes in the resultant displacement vectors of the working medium particles and the machined parts is significantly widened, each particle of working media and the processed part move in different direction vectors, which provides a high probability of collisions at the initial moment of detachment from the walls of the screw rotor 4 , where they have a certain supply of kinetic energy and move with a large kinetic energy, therefore, parts processing intensification is provided.

The speed of the machined parts and working media can be adjusted by the tension spring 12 installed inside the screw rotor, which can ensure the reliability of the longitudinal movement of the load masses inside the screw rotor along its longitudinal axis and discharge into the hopper 9 to receive the processed parts.

Technical and economic advantages arise from the increase in the movement amplitudes, the widening of the range of changes in the resultant displacement vectors of working particles and processed parts, increasing the intensity of their mixing and reorientation, as well as increasing the speed of their movement from loading to unloading, which increases the mixing intensity, increases the energy intensity of interaction between workers particles and machined parts with each other and with the walls of the screw rotor, increases productivity and expands the technological opportunities. However, experiments have shown that the loading masses (machined parts and working medium) in practice are disposed asymmetrically to the axis of rotation of the screw rotor, so there are difficulties in designing such machines and calculating the drive, as well as choosing the optimum power of the motor. Therefore, we developed a technique for calculating the drive of the machine for finishing-cleaning and hardening parts $(\mathrm{OZuUO})$ on the basis of screw rotors. For example, it is specified to provide finishing-cleaning and hardening processing of parts made of steel St3. Steel media are used as working media.

Let us take the average specific gravity of the parts to be processed and the particles of the working media $j_{\mathrm{av} .}=7800 \mathrm{\kappa} \Gamma \mathrm{M}^{3}$.

For example, the screw rotor 4 consists of octahedra, the number of which determines its length. Denoting the edge of the octahedron (Figure 3) - a, the apopheme $-\mathrm{S}$, we 
determine the volume of the octahedron, which can be calculated from formula (1). From the design considerations and the given performance we take the side of the octahedron $a=0.4 \mathrm{~m}$, then the volume of the screw rotor mounted from 9 octahedra will be equal to:

$$
V=9 \cdot a^{3} \cdot \sqrt{2} / 3=0,576 \cdot 0,4714=0,27153 \approx 0,272 \mathrm{M}^{3}
$$

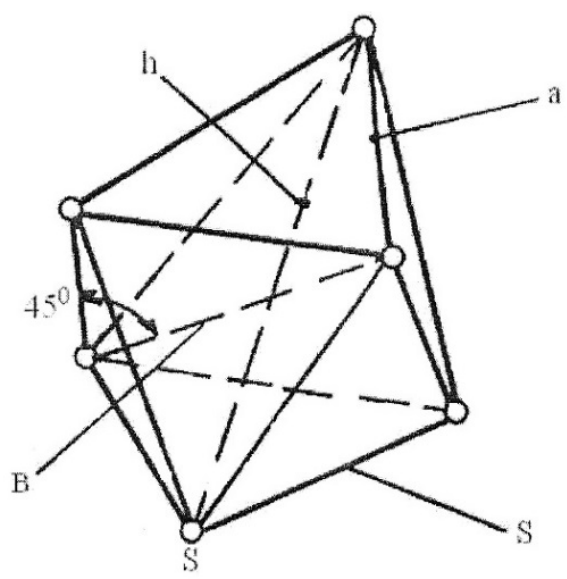

Fig. 3. One of the octahedrons, a visual image.

The number of octahedra is determined by the required constructive technological parameters of the process, namely the length of the screw rotor Lp. and its outer diameter is $\mathrm{Dp}$.

From the condition for filling $K_{v}=0,55$ of the volume of the screw rotor, the mass of machined parts and particles of working media inside the screw rotor can be determined by means of the dependence.

$$
m_{1}=055 \cdot V \cdot \rho
$$

where density $p$ corresponds to the average specific gravity of the machined parts and working medium particles filling the helical rotor. Then:

$$
m_{1}=0,55 \cdot 0,272 \mathrm{~m}^{3} \cdot 7800 \mathrm{~kg} / \mathrm{m}^{3}=1166,88 \mathrm{~kg} \approx 1167 \mathrm{~kg}
$$

To determine the mass of the screw rotor barrel, we calculate its area along the perimeter, which includes 9 octahedra with sides in the form of six equilateral triangles with a side of $0.4 \mathrm{~m}$, i.e. 54 equilateral triangles. And the area of one equilateral triangle is equal to $\mathrm{S}_{1}=a^{2}$. $\sqrt{3} / 4=(1,73 / 4) \cdot a^{2}=0,432 \cdot 0,16 \mathrm{~m}^{2}=0.06912 \mathrm{~m}^{2}$. Then the area of the entire lateral surface of the shaft of the screw rotor will be: $S_{\text {barrel }}=0,06912 \cdot 54=3,73248 \mathrm{~m}^{2} \approx 3,75 \mathrm{~m}^{2}$. Taking for constructive reasons the thickness of the walls of the screw rotor equal to $0.0003 \mathrm{~m}$, we obtain the volume of the metal from which the barrel of the screw rotor is made that is $3,75 \mathrm{~mm}^{2} \cdot 0,003 \mathrm{~m}=0,01125$ and then the mass of the barrel of the screw rotor is equal to:

$$
m_{2}=0,01125 \mathrm{~m}^{3} \cdot 7800 \mathrm{~kg} / \mathrm{m}^{3}=87,75 \mathrm{~kg} \approx 88 \mathrm{~kg} .
$$

Since the diameter of the screw shaft of the screw rotor is determined by the dependence: $D=1.16 \cdot \mathrm{a}=0.464 \mathrm{~m}$, this dimension corresponds to the inner diameter of the rims 5 and 6 (Figure 1). The outer diameters of the rims, for constructive reasons, are 
taken equal to $500 \mathrm{~mm}$. With a rim thickness equal to $0.015 \mathrm{~m}$, the volume of one rim is $0,001635 \mathrm{~m}^{3}$, and the mass of one rim is $m_{3}=13 \mathrm{~kg}$.

In this way:

1. The mass of the parts inside the screw rotor $-m_{1}=1167 \mathrm{~kg}$;

2. The weight of the barrel of the screw rotor $-m_{2}=88 \mathrm{~kg}$;

3 . The mass of two rims rigidly fixed to the outer diameter of the screw rotor $-2 m_{3}=26 \mathrm{~kg}$.

Therefore, provided that the machined parts and working medium particles are evenly distributed, the total mass $m$ of the screw rotor assembly is:

$$
m=m_{1}+m_{2}+2 \cdot m_{3}=1281 \mathrm{~kg} .
$$

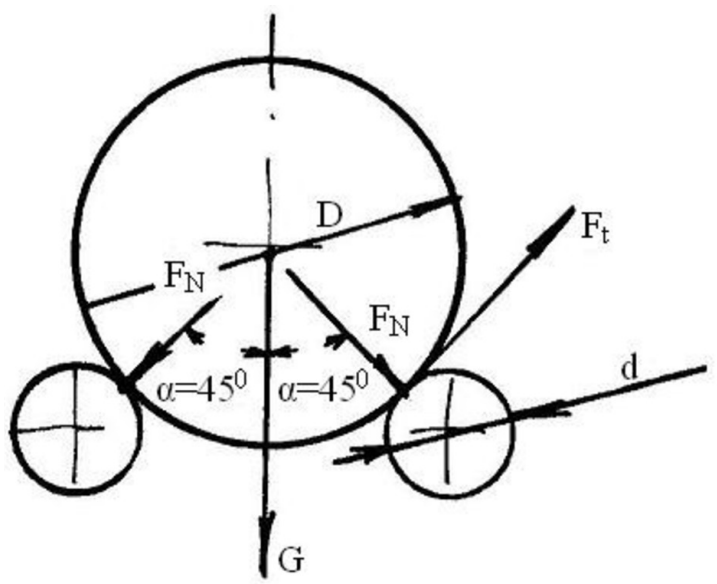

Fig. 4. The scheme of the forces acting in the screw rotor rotation

The force of the screw rotor pressing against the support rollers, provided that the work pieces are evenly distributed in it (Figure 4), can be determined:

$$
\begin{gathered}
F_{N}=\frac{K \cdot F_{t}}{f}=\frac{K \cdot T_{1}(i+1)}{f \cdot D} ; \\
F_{N}=G \cdot \cos \alpha,
\end{gathered}
$$

where $G=1281 \cdot 9,81=12566,6 \mathrm{H}$.

$$
F_{N}=12566,6 \cdot \cos 45^{\circ}=12566,6 \cdot 0,52532 \approx 6601 \mathrm{H}
$$

From this we determine the torque on the support rollers shaft:

$$
\mathrm{M}=\frac{F_{N} \cdot f \cdot D}{K \cdot u}=\frac{6601 \cdot 0,21 \cdot 0,51}{1,3 \cdot 2.5} \approx \frac{707}{3.25} \approx 218 \mathrm{H} . \mathrm{M},
$$

where $\mathrm{f}$ is the friction coefficient, $f=0.21 ; d$ is the drive roller diameter, $d=0.2 \mathrm{~m} ; K$ is the load factor, the reserve of the cord is $K=1.25-1.5$, we assume $K=1.3 ; u$-is the gear ratio from the screw rotor to the support rollers $u=2.5$.

If $n_{p}=70 \mathrm{rev} / \mathrm{min}$ and taking into account that $u=2,5$, the supporting rollers 3 (figure 1) should rotate at a speed of $n=175 \mathrm{rev} / \mathrm{min}$, then:

$$
\begin{gathered}
\omega=\frac{\pi \cdot n}{30}=\frac{3,14 \cdot 175}{30}=18,3\left(\frac{1}{c}\right) ; \\
\eta_{\text {об }}=\eta_{1} \cdot \eta_{2} \cdot \eta_{3}=0,63 ; \\
\eta_{1}=\eta_{3}=0,9 ;
\end{gathered}
$$




$$
\eta_{2}=0,7
$$

In the transmission of the torque $M$ to the roller shaft 3 , a double rotation of the gear and a gearbox takes part, therefore, taking into account the efficiency, the electric motor $N$ in the symmetrical arrangement of the loading of the machined parts and working medium particles, by means of which it is possible to rotate the support rollers 3 and the screw rotor with a total mass $m=1281 \mathrm{~kg}$, can be determined by the dependence:

$$
\mathrm{N}=\frac{\mathrm{M} \cdot \omega}{\eta_{06}}=\frac{218 \cdot 18,3}{0,63}=5332,4 \text { Вт } \approx 5533 \text { Вт. }
$$

In fact, the loading mass of the processed parts and working medium particles being loaded is located in the screw rotor asymmetrically to the axis of rotation. Therefore, it should be assumed that the calculated power of the electric motor for the work of the machine for finishing-cleaning and hardening processing (OZiUO) of the parts will be insufficient. To check this assumption, consider the section of the screw rotor plane perpendicular to the axis of rotation (Fig. 6) with the designation of the quantities used in the calculations, for example, $\varphi$ is the angle of the free surface of the machined parts and working medium particles relative to the horizon, $\alpha$ is the half-angle of the segment of the free volume of the screw rotor, 0 - the center of gravity of the screw rotor loading, $\mathrm{m}_{1}$ - the mass of loading of the processed parts and particles of working media. The radius of ${ }^{\circ} \mathrm{C}$ is denoted by "e" and its value is determined by the formula:

$$
\mathrm{e}=0,424 \cdot\left(1-K_{v}\right) \cdot D=0,424 \cdot 0,45 \cdot 0,5=0,09 \mathrm{~m} .
$$

The torque created relative to the axis of screw rotor rotation by the asymmetrical loading of the machined parts is determined by the dependence:

$$
M_{1}=\mathrm{x}_{\mathrm{e}} \cdot m_{1} \cdot \mathrm{g}=0,055 \cdot 1167 \cdot 9,81=630,18 \approx 631 \mathrm{H} \cdot \mathrm{m},
$$

where $m_{1}$ is the mass of machined parts and particles of working media inside the screw rotor; $\mathrm{x}_{\mathrm{e}}$ - is the projection of the segment $\mathrm{OC}$ on the horizontal axis, determined by the dependence:

$$
\mathrm{x}_{\mathrm{e}}=\mathrm{e} \cdot \sin \varphi=0,095 \cdot 0,5736=0,055 \mathrm{~m} .
$$

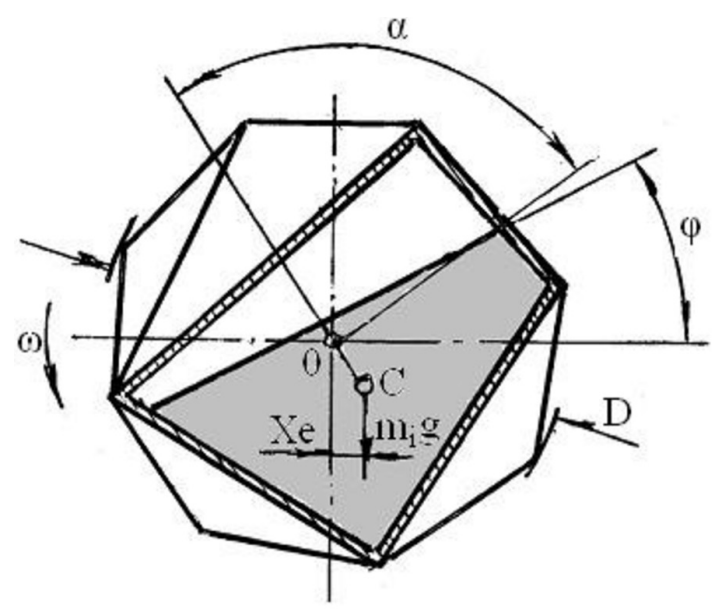

Fig. 5. Scheme for calculating the machine for finishing-cleaning and hardening parts processing (OZiUO) 
Knowing the moment $M_{1}$, created by the loading of the screw rotor, we determine the electric motor's power expended to overcome the moment created by the unbalanced loading of the machined parts relative to the axis of rotation of the screw rotor:

$$
N_{1}=\frac{\mathrm{M} 1 \cdot \omega}{\eta_{\text {об }}}=\frac{631 \cdot 18,3}{0,63}=18329 \mathrm{~W} .
$$

Thus, summing the power of electric motors to rotate the helical rotor from the effects of symmetrical and asymmetric loading, we obtain the value of the total power of the machine's electric motor for finishing and cleaning work on the basis of a screw rotor:

$$
N_{\text {total. }}=N+N_{1}=5533+18329=23862 \mathrm{~W} \approx 24 \mathrm{~kW} .
$$

We select the closest electric motor by reference:

AIR180M4

$N=30 \mathrm{~kW}, \quad n=1500 \mathrm{rev} / \mathrm{min}$.

\section{Conclusions}

As a result of the research:

- The machine is proposed for finishing-cleaning and hardening machining of parts in machine building with a working organ in the form of a screw rotor mounted from hollow octahedra.

- The technique for calculating the drive of the machine for finishing-cleaning and hardening machining of parts in machine building with working bodies in the form of screw rotors mounted from hollow octahedra was created and proposed.

\section{References}

1. V. A. Lebedev, G. V. Serga, A. V. Khandozhko, IOP Conf. Ser.: Mater. Sci. Eng., 327 (2018)

2. G. V. Serga, V. A. Lebedev, Vestnik RGTU im. P. A. Solovyeva, 2 (41), 126 (2017) 